\title{
Effective Control and Bifurcation Analysis in a Chaotic System with Distributed Delay Feedback
}

\author{
Wei Tan, ${ }^{1,2}$ Jianguo Gao, ${ }^{1}$ and Wenjun Fan ${ }^{1}$ \\ ${ }^{1}$ Department of Mathematics and Information Science, Beifang University of Nationalities, Yinchuan, Ningxia 750021, China \\ ${ }^{2}$ Department of Mathematics and Statistics, Zhoukou Normal University, Zhoukou, Henan 466001, China \\ Correspondence should be addressed to Jianguo Gao; gaojguo@163.com
}

Received 21 September 2015; Accepted 26 November 2015

Academic Editor: Ivanka Stamova

Copyright (c) 2016 Wei Tan et al. This is an open access article distributed under the Creative Commons Attribution License, which permits unrestricted use, distribution, and reproduction in any medium, provided the original work is properly cited.

\begin{abstract}
We discuss the dynamic behavior of a new Lorenz-like chaotic system with distributed delayed feedback by the qualitative analysis and numerical simulations. It is verified that the equilibria are locally asymptotically stable when $\alpha \in\left(0, \alpha_{0}\right)$ and unstable when $\alpha \in\left(\alpha_{0}, \infty\right)$; Hopf bifurcation occurs when $\alpha$ crosses a critical value $\alpha_{0}$ by choosing $\alpha$ as a bifurcation parameter. Meanwhile, the explicit algorithm for determining the direction of the Hopf bifurcation and the stability of the bifurcating periodic solutions is derived by normal form theorem and center manifold argument. Furthermore, regarding $\alpha$ as a bifurcation parameter, we explore variation tendency of the dynamics behavior of a chaotic system with the increase of the parameter value $\alpha$.
\end{abstract}

\section{Introduction}

As one of the important discoveries in 21st century, chaos has been extensively investigated in many fields over the last several decades, which has been widely applied in secure communication, signal processing, radar, image processing, power system protection, flow dynamics, and so on. As is known chaos is undesirable and needs to be controlled in many practical applications. Therefore, the investigation of controlling chaos is of great significance. Many schemes have been presented to carry out chaos control [1-17] of which using time-delayed controlling forces proves to be a simple and viable method for a continuous dynamical system.

Recently, a new Lorenz-like system has been introduced in $[18,19]$ as follows:

$$
\begin{aligned}
& \dot{x}(t)=a(y-x), \\
& \dot{y}(t)=b x-l x z, \\
& \dot{z}(t)=-c z+h x^{2}+k y^{2},
\end{aligned}
$$

where $a>0, b>0, c>0, k>0, l>0$, and $h>0$. System (1) exhibits bifurcation and period orbits by taking $h$ as the bifurcation parameter and putting $a=10, b=40, c=2.5$, $l=1$, and $k=2$. In particular, when $h=2$, there appears to be chaotic attractor in [19] (see Figure 3). The conditions of Hopf bifurcation occurring and the stability analysis of the equilibrium points have been studied in detail in [19].

In order to reveal the forming mechanism of the chaotic attractor structure, its controlled system is proposed in [18] as follows:

$$
\begin{aligned}
& \dot{x}(t)=a(y-x), \\
& \dot{y}(t)=b x-l x z+\alpha, \\
& \dot{z}(t)=-c z+h x^{2}+k y^{2},
\end{aligned}
$$

where $a>0, b>0, c>0, k>0, l>0$, and $h>0$. The system exhibits the period-doubling bifurcations taking $e$ as the bifurcation parameter and fixing $a=10, b=40, c=2.5$, $l=1, k=2$, and $h=2$ (see Figure 2). See [18] for more details. In the controller, one can see that when $|e|$ is large enough, chaos attractor disappears and the stable family of limit cycles appears; when $|e|$ is small enough, a complete chaos attractor appears.

With purpose of reflecting and controlling the complex and unpredictable dynamical behavior of the model depending on the past information of the system, it is necessary to incorporate time delay into this system. The signal error of 
current state and past state of the continuous time system will be given distributed delay feedback to the system itself.

As compared with the former method, a chaotic model with distributed delay feedback is more general than that with discrete delay feedback [10-13], because the distributed delay becomes a discrete delay when the delay kernel is a delta function at a certain time. The distributed delay has found widespread applications in many fields such as neural network [14, 16], complicated real models [15], and the modeling of aggregative processes involving the flow of entities with random transit times through a given process [17]. Therefore, it is of considerable significance to propose distributed delays as control input to control the chaotic system.

Many studies have been made in $[18,19]$ about the Lorenzlike system. In this paper, we present the Hopf bifurcation of a Lorenz-like system with the distributed delay. We not only display numerical simulation of Hopf bifurcation in delayed feedback Lorenz-like system, but also give the theoretical proof. The stability of the equilibrium point will vary complicatedly setting different values of the feedback intensity coefficient $m$. Regarding the delay variable $\tau$ as a branch of parameters, when $\tau$ passes through a critical value, the stability of the equilibrium point will change from instability to stability, and then the chaos phenomenon of the system disappears. Finally, the stable periodic solution emerges. Furthermore, flat malicious point of the original system will not change and retain some features of the original system.

Motivated by the above works, we, in this paper, add a time-delayed force $m \int_{-\infty}^{0}[z(t)-z(t+s)] k(-s) d s$ to the third equation of system (1); then system (1) takes the form

$$
\begin{aligned}
\dot{x}(t)= & a(y-x), \\
\dot{y}(t)= & b x-l x z, \\
\dot{z}(t)= & -c z+h x^{2}+k y^{2} \\
& +m \int_{-\infty}^{0}[z(t)-z(t+s)] k(-s) d s,
\end{aligned}
$$

where $a, b, c, h, k, l>0, \int_{-\infty}^{0} k(s) d s=1$, and $\int_{-\infty}^{0} s k(s) d s<$ $\infty$.

The rest of this paper is organized as follows. In Section 2, the stability and the existence of Hopf bifurcation are determined. In Section 3, based on the normal form method and the center manifold theorem presented in Hassard et al. [20], the direction, stability, and the period of the bifurcating period solutions are analyzed. In Section 4, numerical simulations are given to verify the theorem analysis. Finally, Section 5 concludes some discussions.

\section{Bifurcation Analysis of Lorenz-Like System}

In this section, we will study the stability of the equilibria and the existential conditions of local Hopf bifurcations. As the Lorena-like system (3) is symmetric about the $z$-axis, $E_{1}$ and $E_{2}$ have the same stability. It is sufficient to discuss the stability of equilibrium $E_{1}\left(x^{*}, y^{*}, z^{*}\right)$.
By the linear transform

$$
\begin{aligned}
& x_{1}=x-x^{*}, \\
& y_{1}=y-y^{*}, \\
& z_{1}=z-z^{*}
\end{aligned}
$$

system (3) becomes

$$
\begin{aligned}
\dot{x}_{1}(t)= & a\left(y_{1}-x_{1}\right), \\
\dot{y}_{1}(t)= & -b x_{1}-l\left(z^{*} x_{1}+x^{*} z_{1}+x_{1} z_{1}\right), \\
\dot{z}_{1}(t)= & -c z_{1}+h\left(2 x^{*} x_{1}+x_{1}^{2}\right)+k\left(2 y^{*} y_{1}+y_{1}^{2}\right) \\
& +m z_{1}-m \int_{-\infty}^{0}\left[z_{1}(t+s)\right] k(-s) d s .
\end{aligned}
$$

The linearization of (5) near $E\left(x^{*}, y^{*}, z^{*}\right)$ is given by

$$
\begin{aligned}
\dot{x_{1}}(t)= & a\left(y_{1}-x_{1}\right), \\
\dot{y_{1}}(t)= & -b x_{1}-l\left(z^{*} x_{1}+x^{*} z_{1}+x_{1} z_{1}\right), \\
\dot{z_{1}}(t)= & -c z_{1}+h\left(2 x^{*} x_{1}\right)+k\left(2 y^{*} y_{1}\right)+m z_{1} \\
& -m \int_{-\infty}^{0}\left[z_{1}(t+s)\right] k(-s) d s .
\end{aligned}
$$

The Jacobian matrix of (6) at $E_{1}\left(x^{*}, y^{*}, z^{*}\right)$ is written as

$$
J=\left[\begin{array}{ccc}
-a & a & 0 \\
-l z^{*}-b & 0 & -l x^{*} \\
2 h x^{*} & 2 k y^{*} & -c+m-m \int_{-\infty}^{0} k(-s) e^{\lambda s} d s
\end{array}\right],
$$

whose characteristic equation is given by

$$
\begin{aligned}
\lambda^{3}+ & \left(c-m+m \int_{-\infty}^{0} k(-s) e^{\lambda s} d s+a\right) \lambda^{2} \\
& +\left(c-m+m \int_{-\infty}^{0} k(-s) e^{\lambda s} d s\right) a \lambda+2 h l\left(x^{*}\right)^{2} \\
& +a\left(l z^{*}-b\right)\left[\lambda+c-m+m \int_{-\infty}^{0} k(-s) e^{\lambda s} d s\right] \\
& =0 .
\end{aligned}
$$

In this paper, we focus on considering the weak kernel case; that is, $k(s)=\alpha e^{-\alpha s}$, where $\alpha>0$. As to the general gamma kernel case, we can make a similar analysis. We give the initial condition of system (6) as

$$
\left(\begin{array}{l}
x(s) \\
y(s) \\
z(s)
\end{array}\right)=\left(\begin{array}{l}
\phi_{1}(s) \\
\phi_{2}(s) \\
\phi_{3}(s)
\end{array}\right), \quad-\infty<s \leqslant 0 .
$$

The characteristic equation (8) with the weak kernel case takes the form

$$
\lambda^{4}+\theta_{1}(\alpha) \lambda^{3}+\theta_{2}(\alpha) \lambda^{2}+\theta_{3}(\alpha) \lambda+\theta_{4}(\alpha)=0
$$


where

$$
\begin{aligned}
& \theta_{1}(\alpha)=2 a+\alpha-c-m, \\
& \theta_{2}(\alpha)=\alpha(a+c)+2 a(c-m+1)+a\left(l z^{*}-b\right), \\
& \theta_{3}(\alpha)=2 \alpha a c+2 h l\left(x^{*}\right)^{2}+a\left(l z^{*}-b\right)(c-m+\alpha), \\
& \theta_{4}(\alpha)=2 h l \alpha\left(z^{*}\right)^{2}+a c \alpha\left(l z^{*}-b\right) .
\end{aligned}
$$

In view of the well known Routh-Hurwitz criterion, we can conclude that all the roots of (10) have negative real parts if the following conclusions hold:

$$
\begin{aligned}
& D_{1}(\alpha)=\Delta_{1}=\theta_{1}>0, \\
& D_{2}(\alpha)=\Delta_{2}=\theta_{1} \theta_{2}-\theta_{3}>0, \\
& D_{3}(\theta)=\Delta_{3}=\theta_{3} \Delta_{2}-\theta_{4}\left(\theta_{1}\right)^{2}>0, \\
& D_{4}(\theta)=\Delta_{4}=\theta_{4} \Delta_{3}>0 .
\end{aligned}
$$

Based on the analysis above, we can easily obtain the following result.

Theorem 1. The equilibrium $E\left(x^{*}, y^{*}, z^{*}\right)$ of system (3) with the weak kernel is locally asymptotically stable if the following conditions are fulfilled:

$$
\begin{aligned}
& \Delta_{1}=\theta_{1}>0, \\
& \Delta_{2}=\theta_{1} \theta_{2}-\theta_{3}>0, \\
& \Delta_{3}=\theta_{3} \Delta_{2}-\theta_{4}\left(\theta_{1}\right)^{2}>0, \\
& \Delta_{4}=\theta_{4} \Delta_{3}>0 .
\end{aligned}
$$

Let $\lambda_{i}(i=1,2,3,4)$ be the roots of $(10)$; then we have

$$
\begin{aligned}
\sum_{i=1}^{4} \lambda_{i} & =-\theta_{1}(\alpha), \\
\sum_{i=1, j=2, i>j}^{4} \lambda_{i} \lambda_{j} & =\theta_{2}(\alpha), \\
\sum_{i=1, j=2, k=3, i>j>k}^{4} \lambda_{i} \lambda_{k} & =-\theta_{3}(\alpha), \\
\prod_{i=1}^{4} \lambda_{i} & =\theta_{4}(\alpha) .
\end{aligned}
$$

If there exists $\alpha_{0} \in R^{+}$, such that $D_{3}\left(\alpha_{0}\right)=0$ and $\left.\left(d D_{3}(\alpha) / d \alpha\right)\right|_{\alpha=\alpha_{0} \neq 0}$, then, by Routh-Hurwitz criterion, there exists a pair of purely imaginary roots $\lambda_{3}$ and $\lambda_{4}$ that are satisfied: if $\lambda_{3}$ and $\lambda_{4}$ are real, then $\lambda_{3}<0$ and $\lambda_{4}<0$; if $\lambda_{3}$ and $\lambda_{4}$ are complex conjugate, then $\operatorname{Re}\left\{\lambda_{3}\right\}=\operatorname{Re}\left\{\lambda_{4}\right\}=$ $-\operatorname{Re}\left(\theta_{1}(\alpha)\right) / 2$. It is easy to calculate that

$$
\begin{aligned}
& \frac{d\left(\operatorname{Re}\left(\lambda_{1}\right)\right)}{d \alpha} \\
& =-\left.\frac{\theta_{1}(\alpha)}{2\left[\theta_{1}^{3} \theta_{3}(\alpha)+\left(\theta_{1}(\alpha) \theta_{2}(\alpha)-2 \theta_{3}(\alpha)\right)^{2}\right]} \frac{d D_{3}(\alpha)}{d \alpha}\right|_{\alpha=\alpha_{0}},
\end{aligned}
$$

and thus the Hopf bifurcation occurs near $E\left(x^{*}, y^{*}, z^{*}\right)$ when $\alpha$ passes through $\alpha_{0}$.

Theorem 2. Suppose (13) for (10) is satisfied; then system (3) admits the following results:

(i) If $\alpha=\alpha_{0}$, system (3) undergoes a Hopf bifurcation at the equilibria $E_{ \pm}$, respectively.

(ii) If $\alpha \in\left(0, \alpha_{0}\right)$, the equilibria $E_{ \pm}$of system (3) are locally asymptotically stable.

(iii) If $\alpha>\alpha_{0}$, the equilibria $E_{ \pm}$of system (3) are unstable.

Remark 3. It is shown that if (13) is fulfilled, then the states $x, y$, and $z$ of system (1) will tend to $x^{*}, y^{*}$, and $z^{*}$, when $\alpha \in$ $\left(0, \alpha_{0}\right)$. If (13) is satisfied, then the states $x, y$, and $z$ of system (1) may coexist and remain in an oscillatory model near the equilibrium $E\left(x^{*}, y^{*}, z^{*}\right)$. Thus, chaos vanishes, which means that chaos can be controlled.

\section{Direction and Stability of Hopf Bifurcation Period Solution}

In this section, we will establish the explicit formulae determining the direction, the stability, and the period of these periodic solutions bifurcating from the equilibrium $E\left(x^{*}, y^{*}\right.$, $z^{*}$ ) at the critical value of $\alpha_{0}$ by using the normal form theory and the center manifold reduction developed by Hassard et al. [20]. Let $\mu=\alpha-\alpha_{0}$; then system (3) undergoes the Hopf bifurcation at the equilibrium $E\left(x^{*}, y^{*}, z^{*}\right)$ for $\mu=0$. And then $\pm i \omega_{0}$ are purely imaginary roots of the characteristic equation at the equilibrium $E\left(x^{*}, y^{*}, z^{*}\right)$. System (3) can be written as a functional differential equation (FDE) in $C=$ $C\left([-\infty, 0], R^{3}\right)$ as

$$
\dot{u}(t)=A(\mu) u_{t}+R(\mu) u_{t},
$$

where $u(t)=(x(t), y(t), z(t))^{T} \in C$ and $u_{t}=u(t+\theta)=$ $[x(t+\theta), y(t+\theta), z(t+\theta)]^{T} \in C$.

Define

$$
\begin{aligned}
& A(\mu) \phi(\theta) \\
& \quad= \begin{cases}d \frac{\phi(\theta)}{d \theta}, & \theta \in(-\infty, 0), \\
L \phi(\theta)+\int_{-\infty}^{0} F(s) \phi(s), & \theta=0,\end{cases} \\
& R(\mu) \phi(\theta)= \begin{cases}{[0,0,0]^{T},} & \theta \in(-\infty, 0), \\
{\left[0, f_{2}, f_{3}\right]^{T}} & \theta=0,\end{cases}
\end{aligned}
$$

where $\phi(\theta)=\left[\phi_{1}(\theta), \phi_{2}(\theta), \phi_{3}(\theta)\right]^{T} \in C$ and $f_{2}=$ $l \phi_{1}(0) \phi_{3}(0), f_{3}=h \phi_{1}^{2}(0)+k \phi_{2}^{2}(0)$.

For $\psi \in C\left([0,+\infty],\left(R^{3}\right)^{*}\right)$, define

$$
\begin{aligned}
& A^{*} \psi(0) \\
& \quad= \begin{cases}d \frac{\psi(\theta)}{d \theta}, & \theta \in(0,+\infty), \\
L \psi(0)+\int_{-\infty}^{0} F^{T}(t) \psi(-t) d s, & s=0,\end{cases}
\end{aligned}
$$


and define the bilinear inner product

$$
\begin{aligned}
\langle\psi, \phi\rangle= & \overline{\psi(0)} \phi(0) \\
& -\int_{\theta=-1}^{0} \int_{\xi=0}^{\theta} \bar{\psi}^{T}(\xi-\theta) d \eta(\theta) \phi(\xi) d \xi
\end{aligned}
$$

where $\eta(\theta)=\eta(\theta, 0)$. Obviously $A^{*}$ and $A(0)$ are adjoint operator. By the discussion in Section 2, we know that $\pm i \omega_{k} \tau_{k}$ are eigenvalues of $A(0)$. Thus they are eigenvalues of $A^{*}$. We need to calculate the eigenvectors of $A^{*}$ and $A(0)$ corresponding to $-i \omega_{k} \tau_{k}$ and $i \omega_{k} \tau_{k}$, respectively. Let $q(\theta)=(1, \alpha, \beta)^{T} e^{i \theta \omega \tau}$ be the eigenvalues of $A(0)$; that is, $A(0) q(0)=A(\theta)=i \omega_{k} q(\theta)$, and then we have

$$
\begin{aligned}
L q( & (0)+\int_{-\infty}^{0} F(s) q(s) d s \\
= & \left(\begin{array}{ccc}
-a & a & 0 \\
b-l z^{*} & 0 & b-l x^{*} \\
2 h x^{*} & 2 k y^{*} & -c+m
\end{array}\right)\left(\begin{array}{l}
1 \\
\alpha_{1} \\
\alpha_{2}
\end{array}\right) \\
& +\int_{-\infty}^{0}\left(\begin{array}{ccc}
0 & 0 & 0 \\
0 & 0 & 0 \\
0 & 0 & \frac{m \alpha}{i \omega_{0}+\alpha}
\end{array}\right)\left(\begin{array}{l}
1 \\
\alpha_{1} \\
\alpha_{2}
\end{array}\right) e^{i \omega_{0} s} d s \\
= & \left(\begin{array}{c}
-a+a \alpha_{1} \\
2 h x^{*}+2 k y^{*} \alpha_{1}+(m-c) \alpha_{2}+\frac{m \alpha}{i \omega_{0}+\alpha}
\end{array}\right) \\
= & \left(\begin{array}{c}
i \omega_{0} \\
i \omega_{0} \alpha_{1} \\
i \omega_{0} \alpha_{2}
\end{array}\right) \cdot
\end{aligned}
$$

We can obtain

$$
q(\theta)=\left[1, \alpha_{1}, \alpha_{2}\right]^{T} e^{i \omega_{0} \theta}
$$

where

$$
\begin{aligned}
& \alpha_{1}=\frac{i \omega_{0}+a}{a}, \\
& \alpha_{2}=\frac{a\left(b-l z^{*}\right)-i \omega_{0}\left(i \omega_{0}+a\right)}{l x^{*}} .
\end{aligned}
$$

Similarly, assume that $q^{*}(s)=D\left(1, \alpha_{1}^{*}, \alpha_{2}^{*}\right)^{T} e^{i s \omega_{0}}(0 \leqslant s<\infty)$ is the eigenvector of $A^{*}(0)$ corresponding to $-i \omega_{0}$. From the definition of $A^{*}$, we have

$$
q^{*}(s)=D\left[1, \alpha_{1}^{*}, \alpha_{2}^{*}\right]^{T} e^{i \omega_{0} s},
$$

where

$$
\begin{aligned}
& \alpha_{1}^{*}=\frac{\left(i \omega_{0}-a\right) k y^{*}-a h x^{*}}{i \omega_{0} h x^{*}+\left(l z^{*}-b\right) k y^{*}}, \\
& \alpha_{2}^{*}=\frac{-\left(i \omega_{0} \alpha_{1}^{*}+a\right)}{2 k y^{*}} .
\end{aligned}
$$

Since $A^{*}(0) q^{*}(0)=i \omega_{0} q^{*}(0)$, we can have

$$
\begin{aligned}
L^{T} q^{*}(0)+\int_{-\infty}^{0} F^{*}(s) q^{*}(-s) d s \\
=\left(\begin{array}{ccc}
-a & b-l z^{*} & 2 h x^{*} \\
a & 0 & 2 k y^{*} \\
0 & -l x^{*} & -c+m
\end{array}\right)\left(\begin{array}{c}
D \\
D \alpha_{1}^{*} \\
D \alpha_{2}^{*}
\end{array}\right) \\
+\int_{-\infty}^{0}\left(\begin{array}{ccc}
0 & 0 & 0 \\
0 & 0 & 0 \\
0 & 0 & m k(-s)
\end{array}\right)\left(\begin{array}{c}
D \\
D \alpha_{1}^{*} \\
D \alpha_{2}^{*}
\end{array}\right) e^{-i \omega_{0} s} d s \\
=\left(\begin{array}{c}
-a+a \alpha_{1} \\
2 h x^{*}+2 k y^{*} \alpha_{1}+(m-c) \alpha_{2}+\frac{m \alpha}{i \omega_{0}+\alpha}
\end{array}\right) \\
=\left(\begin{array}{c}
-D i \omega_{0} \\
-D i \omega_{0} \alpha_{1}^{*} \\
-D i \omega_{0} \alpha_{2}^{*}
\end{array}\right),
\end{aligned}
$$

where $D$ is a constant such that $\left\langle q^{*}(s), q(\theta)\right\rangle=1$; by (18), we get

$$
\begin{aligned}
& \left\langle q^{*}(s), q(\theta)\right\rangle=\bar{D}\left(1, \overline{\alpha_{1}^{*}}, \overline{\alpha_{2}^{*}}\right)\left(1, \alpha_{1}, \alpha_{2}\right)^{T} \\
& -\int_{-1}^{0} \int_{\xi=0}^{\theta} \bar{D}\left(1, \overline{\alpha_{1}^{*}}, \overline{\alpha_{2}^{*}}\right) e^{-i(\xi-\theta) \omega \tau} d \eta(\theta)\left(1, \alpha_{1}, \alpha_{2}\right)^{T} \\
& \cdot e^{i \xi \omega \tau} d \xi=\bar{D}\left(1+\overline{\alpha_{1}^{*}} \alpha_{1}+\overline{\alpha_{2}^{*}} \alpha_{2}\right) \\
& -\int_{-1}^{0} \int_{\xi=0}^{\theta} \bar{D}\left(1, \overline{\alpha_{1}^{*}}, \overline{\alpha_{2}^{*}}\right) \theta e^{i \theta \omega \tau} d \eta(\theta)\left(1, \alpha_{1}, \alpha_{2}\right)^{T} \\
& =\bar{D}\left(1+\overline{\alpha_{1}^{*}} \alpha_{1}+\overline{\alpha_{2}^{*}} \alpha_{2}+\frac{\alpha m \alpha_{2}^{*} \alpha_{2}}{\alpha-i \omega_{0}}\right)=1 .
\end{aligned}
$$

Therefore, we can choose $D$ as

$$
D=\frac{1}{\left(1+\overline{\alpha_{1}^{*}} \alpha_{1}+\overline{\alpha_{2}^{*}} \alpha_{2}+\alpha m \alpha_{2}^{*} \alpha_{2} /\left(\alpha-i \omega_{0}\right)\right)} .
$$

Next, we employ the idea of Hassrd et al. in [20] to compute the coordinates describing the center manifold coat $\mu=$ 0 . Let $x_{t}$ be the solution of (3) when $\mu=0$.

Defining

$$
\begin{aligned}
z(t) & =\left\langle q^{*}, x_{t}\right\rangle, \\
W(t, \theta) & =x_{t}(\theta)-2 \operatorname{Re}\{z(t) q(\theta)\},
\end{aligned}
$$

on the center manifold $C_{0}$, we get

$$
W(t, \theta)=W(z(t), \overline{z(t)}, \theta),
$$

where

$$
\begin{aligned}
W(t, \theta) & =W(z(t), \overline{z(t)}, \theta)=W(z, \bar{z}) \\
& =W_{20} \frac{z^{2}}{2}+W_{11} z \bar{z}+W_{02} \frac{\bar{z}^{2}}{2}+\cdots,
\end{aligned}
$$


where $z$ and $\bar{z}$ are local coordinates for center manifold $C_{0}$ in the direction of $q^{*}$ and $\overline{q^{*}}$.

Note that $W$ is also real if $x_{t}$ is real. For the solution $x_{t}$, because $\mu=0$, we have

$$
\begin{aligned}
\dot{z}= & \left\langle q^{*}(s), \dot{x}(t)\right\rangle=\left\langle q^{*}(s), A(0) x_{t}+R(0) x_{t}\right\rangle \\
= & \left\langle q^{*}(s), A(0) x_{t}\right\rangle+\left\langle q^{*}(s), R(0) x_{t}\right\rangle \\
& -\int_{-1}^{0} \int_{\xi=0}^{\theta} \overline{q^{*}}(\xi-\theta) \eta(\theta) A(0) R(0) x_{t}(\xi) d(\xi) \\
= & \left\langle i \omega_{0} \tau_{k} q^{*}(s), x_{t}\right\rangle+\overline{q^{*}}(0) f\left(0, x_{t}(\theta)\right) \stackrel{\text { def }}{=} i \omega_{0} z(t) \\
& +\overline{q^{*}}(0)\left[0, f_{2}, f_{3}\right]^{T} .
\end{aligned}
$$

That is,

$$
\dot{z}(t)=i \omega_{0} z+g(z, \bar{z})
$$

where

$$
g(z, \bar{z})=g_{20} \frac{z^{2}}{2}+g_{11} z \bar{z}+g_{02} \frac{\bar{z}^{2}}{2}+\cdots .
$$

Let $f_{0}=\left[0, f_{2}, f_{3}\right]^{T}$; we have

$$
\begin{aligned}
g(z, \bar{z}) & =\overline{q^{*}(0)} f_{0}(z, \bar{z})=f\left(0, x_{t}\right) \\
& =\bar{D}\left(1, \overline{\alpha_{1}^{*}}, \overline{\alpha_{2}^{*}}\right)\left(0, f_{2}\left(0, x_{t}\right), f_{3}\left(0, x_{t}\right)\right)^{T} .
\end{aligned}
$$

Comparing the coefficients of (33) with (34), we get

$$
\begin{aligned}
g_{20} & =2 \bar{D}\left[l \alpha_{2} \alpha_{1}^{*}+\overline{\alpha_{2}^{*}}\left(h+k \alpha_{1}^{2}\right)\right], \\
g_{11} & =\bar{D}\left[l \alpha_{1}^{*}\left(\alpha_{2}+\overline{\alpha_{2}}\right)+\overline{\alpha_{2}^{*}}\left(2 h+2 k \alpha_{1} \overline{\alpha_{1}}\right)\right], \\
g_{02} & =2 \bar{D}\left[l \overline{\alpha_{1}^{*}} \overline{\alpha_{2}}+\overline{\alpha_{2}^{*}}\left(2 h+2 k \alpha_{1} \overline{\alpha_{1}}\right)\right], \\
g_{21} & =2 \bar{D}\left[l \overline { \alpha _ { 1 } ^ { * } } \left(W_{11}^{(3)}(0)+\frac{1}{2} W_{20}^{(3)}(0)+\frac{1}{2} \overline{\alpha_{2}} W_{20}^{(1)}(0)\right.\right. \\
& \left.+\frac{1}{2} \alpha_{2} W_{11}^{(1)}(0)\right)+\overline{\alpha_{2}^{*}}\left(2 h W_{11}^{(1)}+h W_{20}^{(1)}+k \alpha_{1} W_{11}^{(2)}\right. \\
& \left.+k \overline{\alpha_{1}} W_{20}^{(2)}\right)+\frac{1}{2} \overline{\alpha_{1}} W_{11}^{(3)}(0)+\frac{1}{2} \overline{\alpha_{2}} W_{11}^{(2)}(0) \\
& \left.\cdot \overline{\alpha_{1}^{*}} W_{20}^{(1)}(0)+2 \overline{\alpha_{1}^{*}} W_{11}^{(1)}(0)\right] .
\end{aligned}
$$

Since $W_{20}^{(1)}(0), W_{20}^{(3)}(0), W_{11}^{(1)}(0), W_{11}^{(2)}(0)$, and $W_{11}^{(3)}(0)$ in $g_{21}$ are unknown, we still need to compute them, so we have

$$
\begin{aligned}
& \dot{W}= \begin{cases}A W-2 \operatorname{Re}\left\{\overline{q^{*}(0)}\left[0, f_{2}, f_{3}\right]^{T} q(\theta)\right\}, & \theta \in[-1,0) \\
A W-2 \operatorname{Re}\left\{\overline{q^{*}(0)}\left[0, f_{2}, f_{3}\right]^{T} q(\theta)\right\}+\left[0, f_{2}, f_{3}\right]^{T}, & \theta=0,\end{cases} \\
& \stackrel{\text { def }}{=} A W+H(z, \bar{z}, \theta) .
\end{aligned}
$$

We can rewrite

$$
\dot{W}=A W+H(z, \bar{z}, \theta),
$$

where

$$
H(z, \bar{z}, \theta)=H_{20} \frac{z^{2}}{2}+H_{11} z \bar{z}+H_{02} \frac{\bar{z}^{2}}{2}+H_{21} \frac{z^{2} \bar{z}}{2} \cdots .
$$

Comparing the coefficients, we get

$$
\begin{aligned}
\left(A-2 i \omega_{0}\right) W_{20}(\theta) & =-H_{20}, \\
A W_{11}(\theta) & =-H_{11}(\theta) .
\end{aligned}
$$

For $\theta \in[-\infty, 0)$,

$$
\begin{aligned}
H(z, \bar{z}, \theta) & =-\overline{q^{*}}(0) f_{0} q(\theta)-q^{*}(0) \overline{f_{0} q(\theta)} \\
& =-g(z, \bar{z}) q(\theta)-\bar{g}(z, \bar{z}) \bar{q}(\theta),
\end{aligned}
$$

comparing the coefficients of (38) with (40), gives

$$
\begin{aligned}
& H_{20}(\theta)=-g_{20} q(\theta)-\bar{g}_{02} \bar{q}(\theta), \\
& H_{11}(\theta)=-g_{11} q(\theta)-\bar{g}_{11} \bar{q}(\theta) .
\end{aligned}
$$

By (39) and (41) and the definition of $A$, we obtain

$$
\dot{W}_{20}=2 i \omega_{0} \tau_{k} W_{20}(\theta)+g_{20} q(\theta)+\bar{g}_{02} \bar{q}(\theta) .
$$

Since $q(\theta)=q(0) e^{i \omega_{0} \tau_{k} \theta}$, we get

$$
\begin{aligned}
W_{20}(\theta)= & \frac{i g_{20}}{\omega_{0} \tau_{k}} q(0) e^{i \omega_{0} \tau_{k} \theta}+\frac{i \bar{g}_{02}}{3 \omega_{0} \tau_{k}} \bar{q}(0) e^{-i \omega_{0} \tau_{k} \theta} \\
& +E_{1} e^{2 i \omega_{0} \theta},
\end{aligned}
$$

where $E_{1}=\left(E_{1}^{(1)}, E_{1}^{(2)}, E_{1}^{(3)}\right)^{T}$ is a constant vector.

Similarly, in view of (39) and (41) and the definition of $A$ we get

$$
\begin{aligned}
\dot{W}_{11}(\theta)= & g_{11} q(\theta)+\bar{g}_{11} \bar{q}(\theta), \\
W_{11}(\theta)= & -\frac{i g_{11}}{\omega_{0} \tau_{k}} q(0) e^{i \omega_{0} \tau_{k} \theta}+\frac{i \bar{g}_{11}}{\omega_{0} \tau_{k}} \bar{q}(0) e^{-i \omega_{0} \tau_{k} \theta} \\
& +E_{2},
\end{aligned}
$$

where $E_{1}=\left(E_{2}^{(1)}, E_{2}^{(2)}, E_{2}^{(3)}\right)^{T}$ is a constant vector. 
Next, we will seek appropriate $E_{1}, E_{2}$ in (43) and (45), respectively; by the definition of $A$ and (41),

we have

$$
\begin{aligned}
& \dot{W}_{20}(\theta)=\int_{-1}^{0} d \eta(\theta) W_{20}(\theta)=2 i \omega_{0} \tau_{k} W_{20}-H_{20}(0), \\
& \dot{W}_{11}(\theta)=\int_{-1}^{0} d \eta(\theta) W_{11}(\theta)=-H_{11}(0)
\end{aligned}
$$

where $\eta(\theta)=\eta(0, \theta)$. By (41), we get

$$
\begin{aligned}
H_{20}(\theta)= & -g_{20} q(\theta)-\bar{g}_{02} \bar{q}(\theta)+2\left[0, l \alpha_{2}, h+k \alpha_{1}^{2}\right]^{T} \\
H_{11}(\theta)= & -g_{11} q(\theta)-\bar{g}_{11} \bar{q}(\theta) \\
& +2\left[0, l\left(\alpha_{2}+\overline{\alpha_{2}}\right), 2 h+2 k \alpha_{1} \bar{\alpha}_{1}\right]^{T}
\end{aligned}
$$

For $i \omega_{k}$ is the eigenvalues of $A(0)$ and $q(0)$ is the corresponding eigenvector, we obtain

$$
\begin{gathered}
\left(i \omega_{k}-\int_{-1}^{0} e^{i \theta \omega \tau} d \eta(\theta)\right) q(0)=0, \\
\left(-i \omega_{k}-\int_{-1}^{0} e^{-i \theta \omega} d \eta(\theta)\right) \overline{q(0)}=0 .
\end{gathered}
$$

Substituting (43) and (48) into (46), we obtain

$$
\left(2 i \omega_{k}-\int_{-1}^{0} e^{2 i \theta \omega} d \eta(\theta)\right) E_{1}=2\left[0, l \alpha_{2}, h+k \alpha_{1}^{2}\right]^{T}
$$

which is equivalent to

$$
\begin{aligned}
& \left(\begin{array}{ccc}
2 i \omega_{0}+a & l z^{*}-B & -2 h x^{*} \\
-a & 2 i \omega_{0} & -2 k y^{*} \\
0 & l Z^{*} & 2 i \omega_{0}-m+c+\frac{m \alpha}{\alpha+2 i \omega_{0}}
\end{array}\right) E_{1} \\
& =\left(\begin{array}{c}
0 \\
l \alpha_{2} \\
h+k \alpha_{1}^{2}
\end{array}\right)
\end{aligned}
$$

It follows that

$$
\begin{aligned}
& E_{1}^{(1)}=\frac{\Delta_{11}}{\Delta_{1}}, \\
& E_{1}^{(2)}=\frac{\Delta_{12}}{\Delta_{1}}, \\
& E_{1}^{(3)}=\frac{\Delta_{13}}{\Delta_{1}},
\end{aligned}
$$

where

$\Delta_{11}$

$$
=2\left(\begin{array}{ccc}
0 & l z^{*}-b & -2 h x^{*} \\
l \alpha_{2} & 2 i \omega_{0} & -2 k y^{*} \\
h+k \alpha_{1}^{2} & l z^{*} & 2 i \omega_{0}-m+c+\frac{m \alpha}{\alpha+2 i \omega_{0}}
\end{array}\right),
$$

$\Delta_{12}$

$=2\left(\begin{array}{ccc}2 i \omega_{0}+a & 0 & -2 h x^{*} \\ -a & l \alpha_{2} & -2 k y^{*} \\ 0 & h+k \alpha_{1}^{2} & 2 i \omega_{0}-m+c+\frac{m \alpha}{\alpha+2 i \omega_{0}}\end{array}\right)$,

$\Delta_{13}=2\left(\begin{array}{ccc}2 i \omega_{0}+a & l z^{*}-b & 0 \\ -a & 2 i \omega_{0} & l \alpha_{2} \\ 0 & l z^{*} & h+k \alpha_{1}^{2}\end{array}\right)$,

$\Delta_{1}$

$=\left(\begin{array}{ccc}2 i \omega_{0}+a & l z^{*}-b & -2 h x^{*} \\ -a & 2 i \omega_{0} & -2 k y^{*} \\ 0 & l z^{*} & 2 i \omega_{0}-m+c+\frac{m \alpha}{\alpha+2 i \omega_{0}}\end{array}\right)$.

Similarly, substituting (44) and (49) into (47), we have

$$
\begin{aligned}
& \left(\begin{array}{ccc}
-a & b-l z^{*} & 2 h x^{*} \\
a & 0 & 2 k y^{*} \\
0 & -l z^{*} & m-c+\frac{m \alpha}{\alpha+i \omega_{0}}
\end{array}\right) E_{2} \\
& =2\left(\begin{array}{c}
l\left(\alpha_{2}+\overline{\alpha_{2}}\right) \\
2 h+2 k \alpha_{1} \overline{\alpha_{1}}
\end{array}\right), \\
& E_{2}^{(1)}=\frac{\Delta_{21}}{\Delta_{2}}, \\
& E_{2}^{(2)}=\frac{\Delta_{22}}{\Delta_{2}},
\end{aligned}
$$$$
E_{2}^{(3)}=\frac{\Delta_{23}}{\Delta_{2}}
$$

$\Delta_{21}$

$$
=2\left(\begin{array}{ccc}
0 & b-l z^{*} & 2 h x^{*} \\
l\left(\alpha_{2}+\overline{\alpha_{2}}\right) & 0 & 2 k y^{*} \\
2 h+2 k \alpha_{1} \overline{\alpha_{1}} & -l z^{*} & m-c+\frac{m \alpha}{\alpha+i \omega_{0}}
\end{array}\right),
$$




$$
\begin{aligned}
& \Delta_{22}=2\left(\begin{array}{ccc}
-a & 0 & 2 h x^{*} \\
a & l\left(\alpha_{2}+\overline{\alpha_{2}}\right) & 2 k y^{*} \\
0 & 2 h+2 k \alpha_{1} \overline{\alpha_{1}} & m-c+\frac{m \alpha}{\alpha+i \omega_{0}}
\end{array}\right), \\
& \Delta_{23}=2\left(\begin{array}{ccc}
-a & b-l z^{*} & 0 \\
a & 0 & l\left(\alpha_{2}+\overline{\alpha_{2}}\right) \\
0 & -l z^{*} & 2 h+2 k \alpha_{1} \overline{\alpha_{1}}
\end{array}\right), \\
& \Delta_{2}=\left(\begin{array}{ccc}
-a & b-l z^{*} & 2 h x^{*} \\
a & 0 & 2 k y^{*} \\
0 & -l z^{*} & m-c+\frac{m \alpha}{\alpha+i \omega_{0}}
\end{array}\right) .
\end{aligned}
$$

Consequently, we can determine $W_{20}(0)$ and $W_{11}(0)$. Thus, all $g_{i j}$ can be determined by [7]. Following the basic idea of [7] and the method in [20], one can draw the conclusion about the bifurcation direction and the stability of the Hopf bifurcation, which are determined by the following parameters:

$$
\begin{aligned}
C_{1}(0) & =\frac{i}{2 \omega_{0}}\left(g_{20} g_{11}-2\left|g_{11}\right|^{2}-\frac{1}{3}\left|g_{02}\right|^{2}\right)+\frac{g_{21}}{2}, \\
\mu_{2} & =-\frac{\operatorname{Re}\left\{C_{1}(0)\right\}}{\operatorname{Re}\left\{\lambda^{\prime}\left(\alpha_{0}\right)\right\}} \\
T_{2} & =\frac{\operatorname{Im}\left\{C_{1}(0)\right\}+\mu_{2} \operatorname{Im}\left\{\lambda^{\prime}\left(\alpha_{0}\right)\right\}}{\omega_{0}}, \\
\beta_{2} & =2 \operatorname{Re}\left\{C_{1}(0)\right\}
\end{aligned}
$$

which determines the quantities of bifurcating periodic solutions on the center manifold $C_{0}$; namely, we have the following result.

Theorem 4. $\mu_{2}$ determines the direction of the Hopf bifurcation, if $\mu_{2}>0\left(\mu_{2}<0\right)$; $\beta_{2}$ determines the stability of the bifurcating period solutions: the bifurcation period solutions are orbitally stable (unstable) if $\beta_{2}<0\left(\beta_{2}>0\right)$, and $T_{2}$ determines the period of the bifurcating periodic solutions: the period increases (decreases) if $T_{2}>0\left(T_{2}<0\right)$.

\section{Computer Simulations}

In this section, we give numerical simulation result of system (3) to certify our theoretical analysis. Let us consider the following system:

$$
\begin{aligned}
\dot{x}(t)= & 10(y-x), \\
\dot{y}(t)= & 40 x-x z, \\
\dot{z}(t)= & -2.5 z+2 x^{2}+2 y^{2} \\
& +m \int_{-\infty}^{0}[z(t)-z(t+s)] k(-s) d s,
\end{aligned}
$$

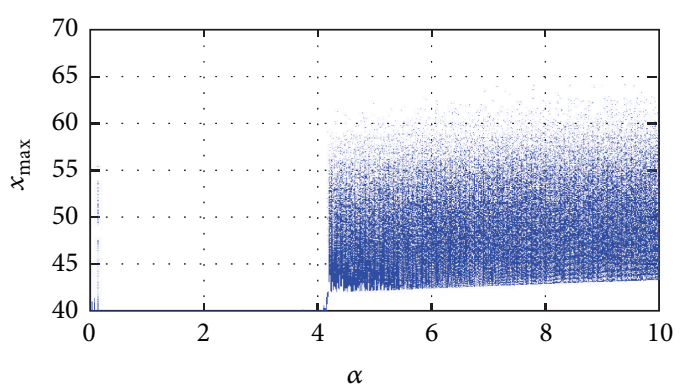

FIGURE 1: The bifurcation diagrams of system (2) with $a=10, b=40$, $c=2.5, k=2, h=2$, and $l=1$.

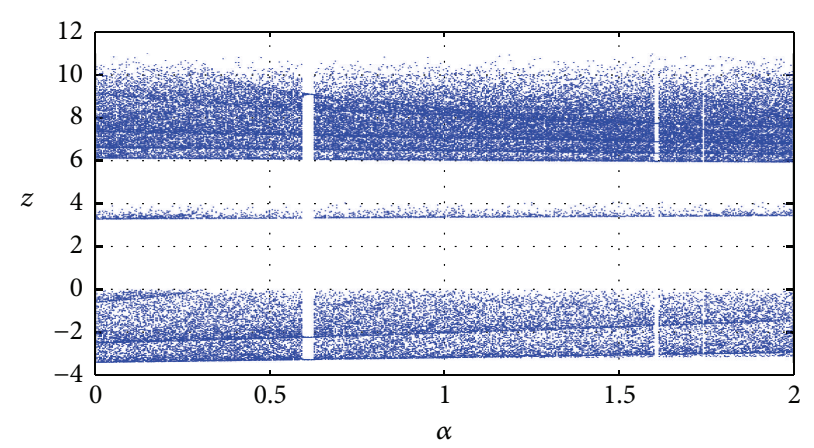

FIGURE 2: The bifurcation diagrams of system (2) with $a=10, b=$ $40, c=2.5, k=2, h=2$, and $l=1$.

where $k(s)=\alpha e^{-\alpha s}, \alpha>0$. By simple calculation, we know (57) has three equilibrium points $E_{0}(0,0,0), E_{1}(5,5,40)$, and $E_{2}(-5,-5,40)$. For equilibrium point $E_{0}(0,0,0)$ will be stable. Furthermore, we conclude that the bifurcating periodic solution is also stable in the phase space even though it is stable in the center manifold. From the equilibria $E_{1}(5,5,40)$, $E_{2}(-5,-5,40)$ all the conditions indicated in Theorem 4 are satisfied. By means of Matlab 7.8.0, we get $\alpha_{0}=4.1$. Thus the equilibria $E_{1}(5,5,40), E_{2}(-5,-5,40)$ are asymptotically stable when $\alpha<\alpha_{0}=4.1$ which is illustrated by the computer simulations (see Figures 1, 3, 4, and 5). When $\alpha$ passes through the critical value $\alpha_{0}$, the equilibria $E_{1}(5,5,40), E_{2}(-5,-5,40)$ lose their stability and Hopf bifurcation occurs (see Figure 6) and quasi-periodic solutions appear (see Figure 7). With increasing of $\alpha$, the bifurcation numerical simulations show the bifurcating quasi-periodic solutions disappear when $\alpha>$ $\alpha_{0}$, and chaos occurs again (see Figures 1 and 8 ).

Remark 5. Since the original system (1) is chaotic, there is no stabilized orbit. When we add distributed delayed feedback perturbations to the original system (1), then, under some suitable condition, stabilized orbits will occur. Thus, we can conclude that the stabilized orbits of the original system (1) are delay-induced.

\section{Conclusion}

In this paper, we investigate a Lorenz-like system within chaotic attractor responding to the local Hopf bifurcation and 

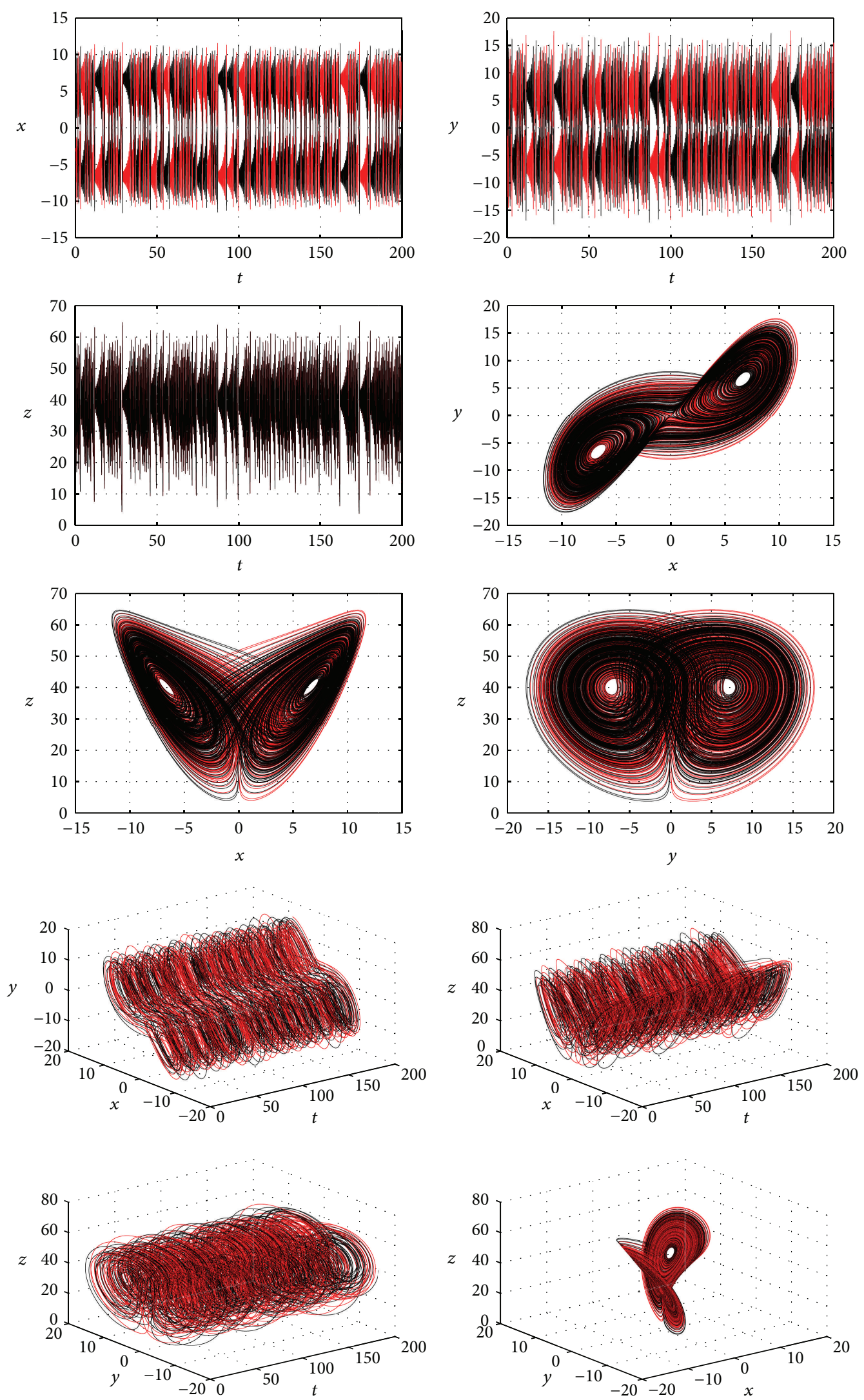

Figure 3: The chaotic attractors of system (3) with $a=10, b=40, c=2.5, k=2, h=2$, and $l=1$. 

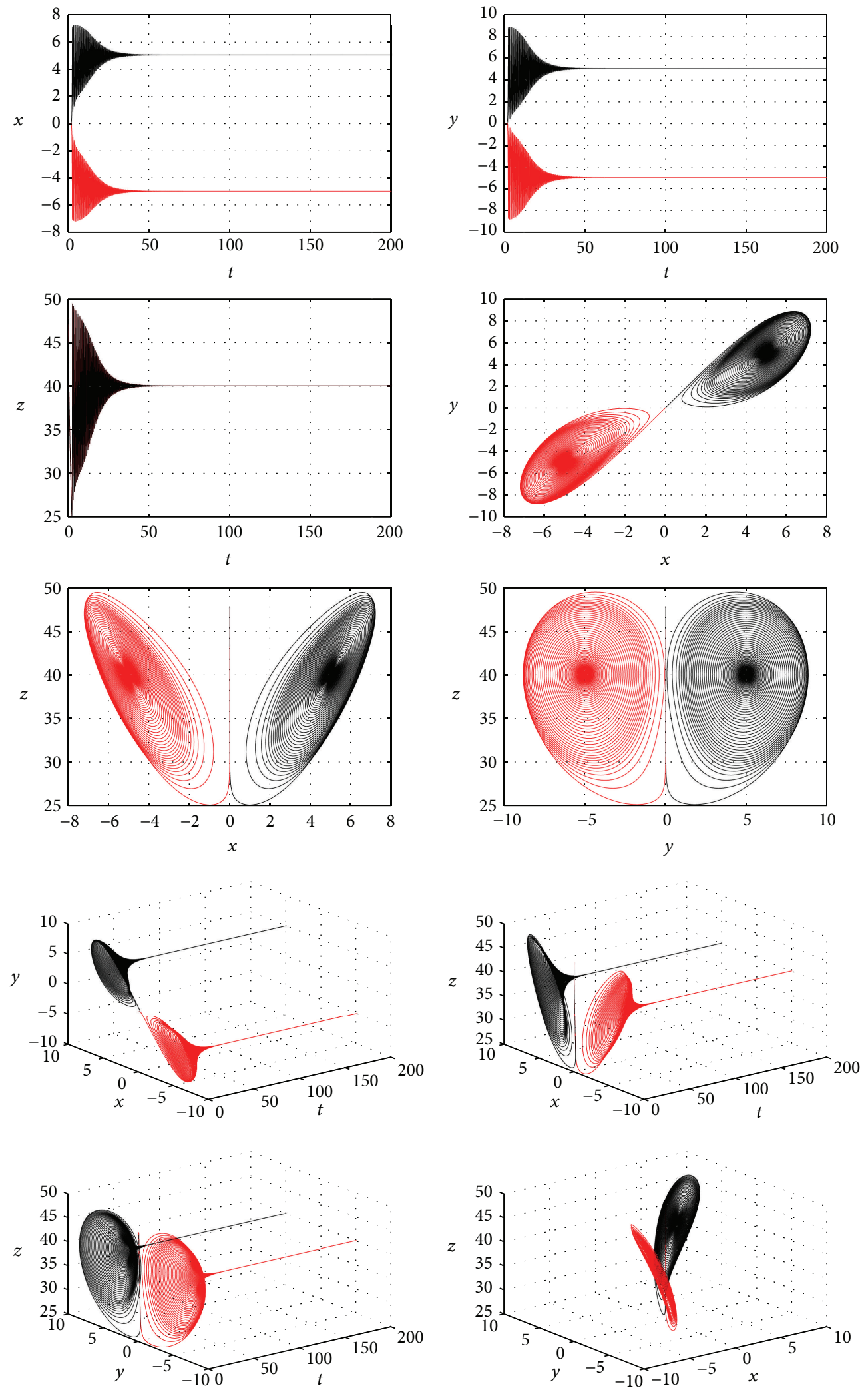

FIGURE 4: Behavior and phase portraits of system (3) with $a=10, b=40, c=2.5, k=2, h=2, l=1, m=-2$, and $\alpha=0.5<\alpha_{0}$. The equilibria $E_{1}$ (red) and $E_{2}$ (black) are asymptotically stable. 

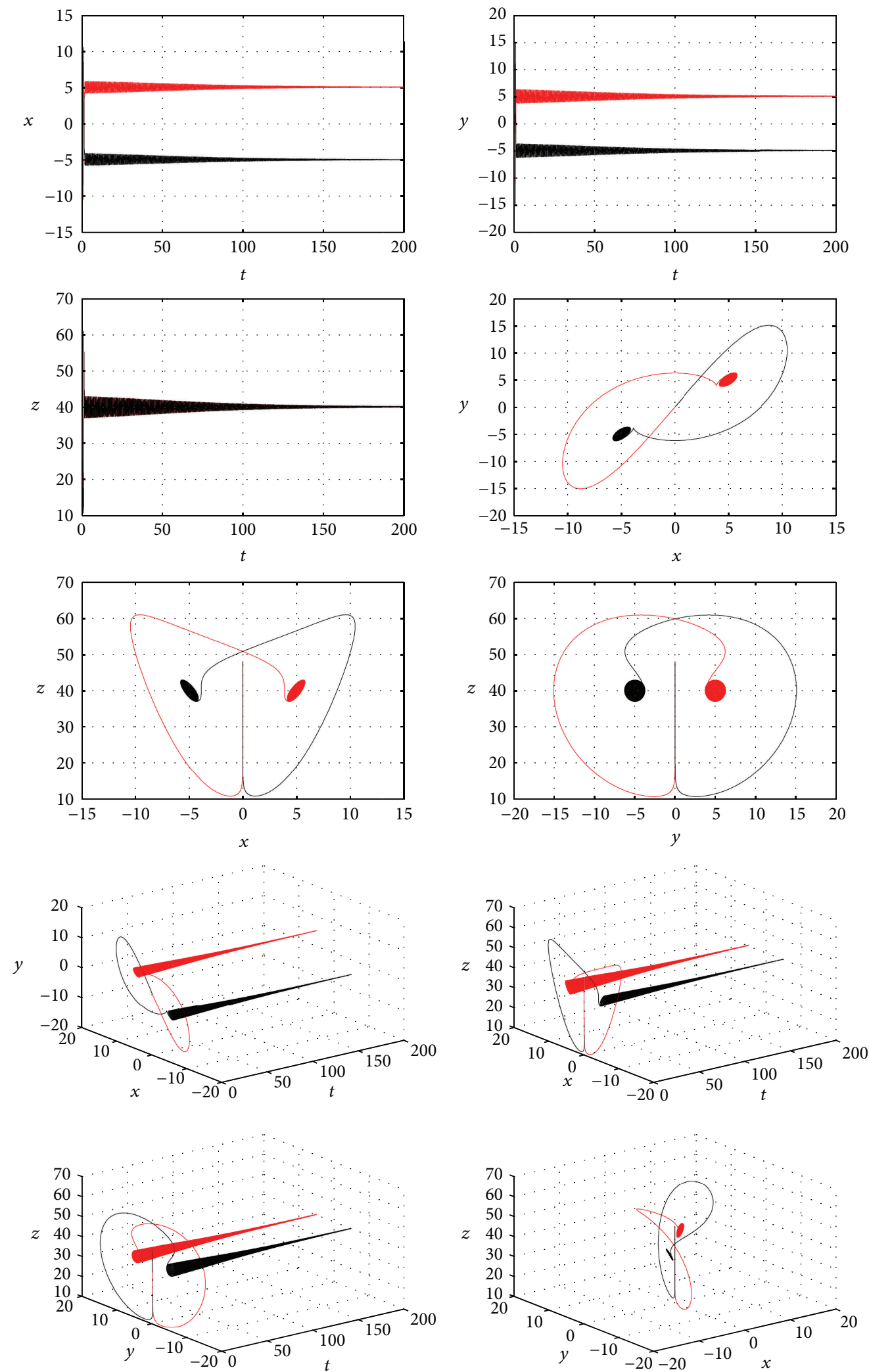

Figure 5: Behavior and phase portraits of system (3) with $a=10, b=40, c=2.5, k=2, h=2, l=1$, and $\alpha=3.95<\alpha_{0}$. The equilibria $E_{1}$ (red) and $E_{2}$ (black) are asymptotically stable. 

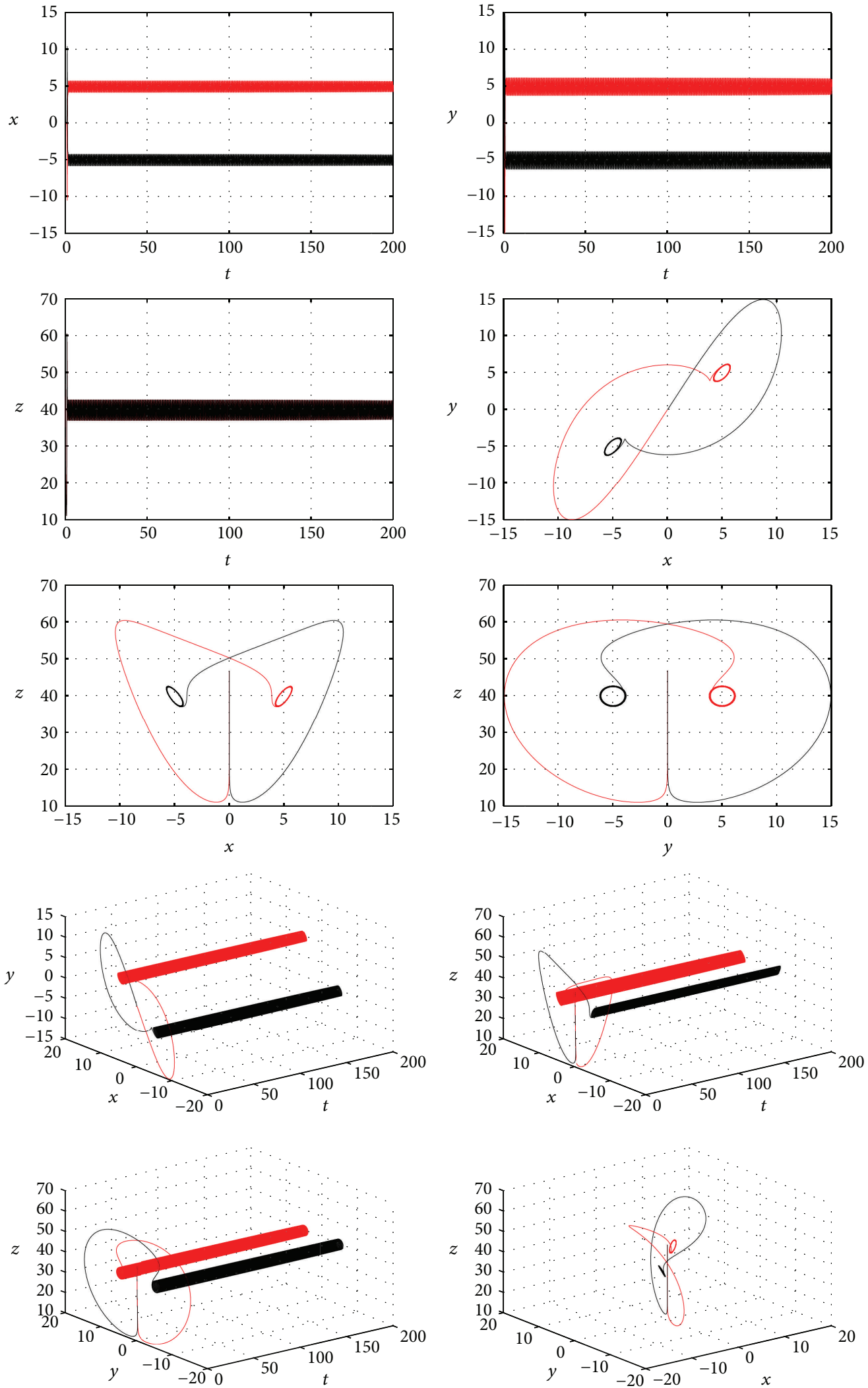

FIGURE 6: Behavior and phase portraits of system (3) with $a=10, b=40, c=2.5, k=2, h=2, l=1$, and $\alpha=4.1=\alpha_{0}$. The equilibria $E_{1}$ (red) and $E_{2}$ (black) are asymptotically stable. 

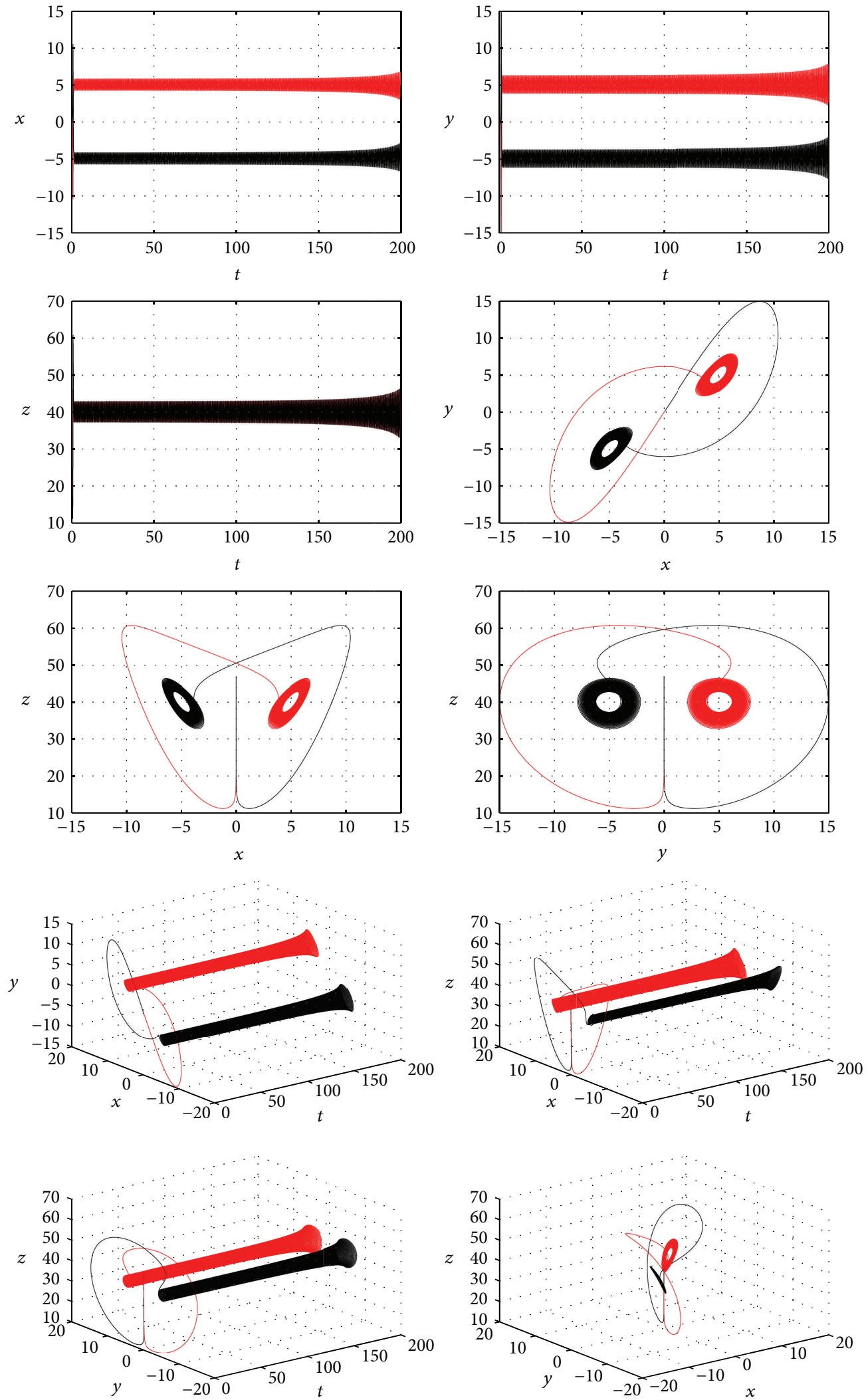

Figure 7: The quasi-period solution of system (3) with $a=10, b=40, c=2.5, m=-2, k=2, h=2, l=1, m=-2$, and $\alpha=4.103>\alpha_{0}$. The equilibria $E_{1}$ (red) and $E_{2}$ (black) are asymptotically stable. 

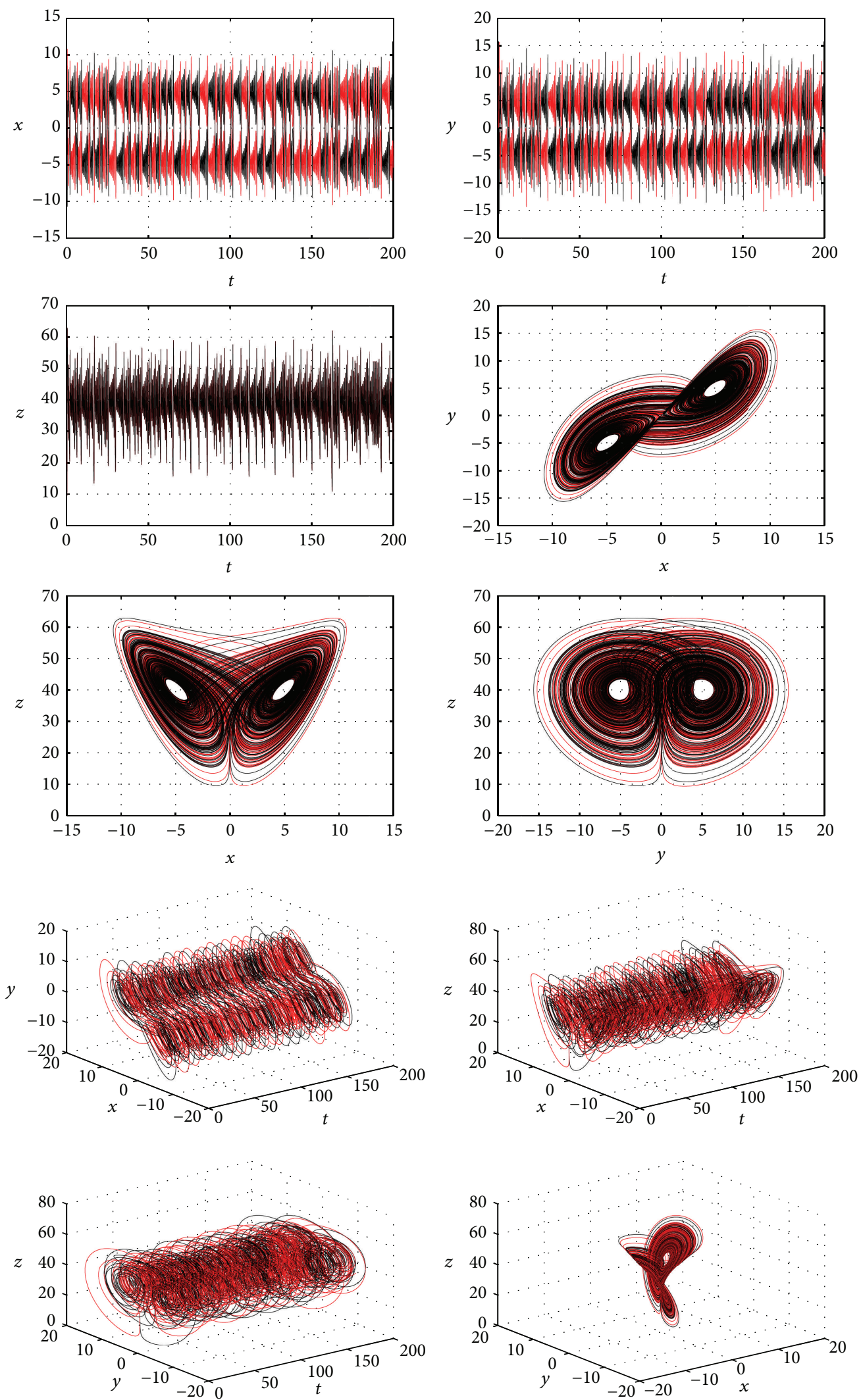

Figure 8: The chaotic attractor of system (3) with $a=10, b=40, c=2.5, k=2, h=2, l=1, m=-2$, and $\alpha=10>\alpha_{0}$; chaos again exists. 
the local stability of equilibrium $E_{ \pm}$. Meanwhile, we study the direction of Hopf bifurcation and the stability of bifurcating periodic solutions by using the center manifold theorem and normal form method. Numerical simulation results confirm that the new feedback controller using distributed delay feedback is an effective method for chaos control. The results show the periodic solutions disappear and chaos attractor appears again with $\alpha$ increasing. Through further investigation, we expect that the chaos system and physicists can decide on which bifurcation arises in the chaos model by proper setting on the feedback parameters for building programs to suppress chaos. In addition, it will be pointed out that the real time evolutions of the chaos systems show marked discrete feature due to their small systems sizes. It is interesting for us to discuss the Lorenz-like system reaction dynamics of discrete Lorenz-like system systems. And it will be further investigated elsewhere in the future.

\section{Conflict of Interests}

The authors declare that there is no conflict of interests regarding the publication of this paper.

\section{Acknowledgments}

This work was supported by National Natural Science Foundation of China (Grant no. 61261044) and the Natural Science and Technology Foundation of Henan Province (no. 15A110046).

\section{References}

[1] C. J. Xu and Y. S. Wu, "Chaos control and bifurcation behavior for a Sprott E system with distributed delay feedback," International Journal of Automation and Computing, vol. 12, no. 2, pp. 182-191, 2015.

[2] C. J. Xu and Q. M. Zhang, "On the chaos control of the Qi system," Journal of Engineering Mathematics, vol. 90, no. 1, pp. 67-81, 2015

[3] E.-W. Bai and K. E. Lonngren, "Sequential synchronization of two Lorenz systems using active control," Chaos, Solitons and Fractals, vol. 11, no. 7, pp. 1041-1044, 2000.

[4] G. R. Chen and X. Dong, "On feedback control of chaotic continuous-time systems," IEEE Transactions on Circuits and Systems I: Fundamental Theory and Applications, vol. 40, no. 9, pp. 591-601, 1993.

[5] H. N. Agiza, "Controlling chaos for the dynamical system of coupled dynamos," Chaos, Solitons \& Fractals, vol. 13, no. 2, pp. 341-352, 2002.

[6] J. A. K. Suykens, P. F. Curran, and L. O. Chua, "Master-slave synchronization using dynamic output feedback," International Journal of Bifurcation and Chaos in Applied Sciences and Engineering, vol. 7, no. 3, pp. 671-679, 1997.

[7] K. Pyragas, "Continuous control of chaos by self-controlling feedback," Physics Letters A, vol. 170, no. 6, pp. 421-428, 1992.

[8] M. T. Yassen, "Chaos control of Chen chaotic dynamical system," Chaos, Solitons \& Fractals, vol. 15, no. 2, pp. 271-283, 2003.

[9] Q.-L. Han, "On designing time-varying delay feedback controllers for master-slave synchronization of Lur'e systems," IEEE
Transactions on Circuits and Systems. I. Regular Papers, vol. 54, no. 7, pp. 1573-1583, 2007.

[10] Y. Song and J. Wei, "Bifurcation analysis for Chen's system with delayed feedback and its application to control of chaos," Chaos, Solitons \& Fractals, vol. 22, no. 1, pp. 75-91, 2004.

[11] S. Guo, G. Feng, X. Liao, and Q. Liu, "Hopf bifurcation control in a congestion control model via dynamic delayed feedback," Chaos, vol. 18, no. 4, Article ID 043104, 2008.

[12] S. Gakkhar and A. Singh, "Complex dynamics in a prey predator system with multiple delays," Communications in Nonlinear Science and Numerical Simulation, vol. 17, no. 2, pp. 914-929, 2012.

[13] F. M. Atay, "Delayed feedback control near Hopf bifurcation," Discrete and Continuous Dynamical Systems Series S, vol. 1, no. 2, pp. 197-205, 2008.

[14] L. Zhou and F. Chen, "Hopf bifurcation and Si'lnikov chaos of Genesio system," Chaos, Solitons \& Fractals, vol. 40, no. 3, pp. 1413-1422, 2009.

[15] K. Balachandran, Y. Zhou, and J. Kokila, "Relative controllability of fractional dynamical systems with delays in control," Communications in Nonlinear Science and Numerical Simulation, vol. 17, no. 9, pp. 3508-3520, 2012.

[16] H. Zhao and L. Wang, "Hopf bifurcation in Cohen-Grossberg neural network with distributed delays," Nonlinear Analysis: Real World Applications, vol. 8, no. 1, pp. 73-89, 2007.

[17] T. J. Manetsch, "Time-varying distributed delays and their use in aggregative models of large systems," IEEE Transactions on Systems, Man and Cybernetics, vol. 6, no. 8, pp. 547-553, 1976.

[18] L. Chongxin, L. Ling, L. Tao, and L. Peng, "A new butterflyshaped attractor of Lorenz-like system," Chaos, Solitons and Fractals, vol. 28, no. 5, pp. 1196-1203, 2006.

[19] R. Wu and T. Fang, "Stability and Hopf bifurcation of a Lorenzlike system," Applied Mathematics and Computation, vol. 262, pp. 335-343, 2015.

[20] B. Hassard, N. Kazarinoff, and Y. Wan, Theorem and Application of Hopf Bifurcation, Cambridge University Press, Cambridge, UK, 1981. 


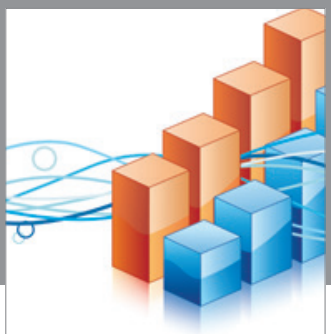

Advances in

Operations Research

vatem alat4

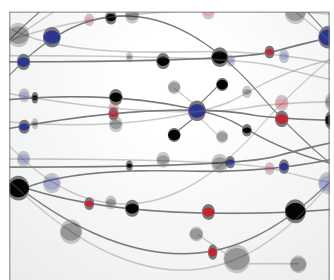

\section{The Scientific} World Journal
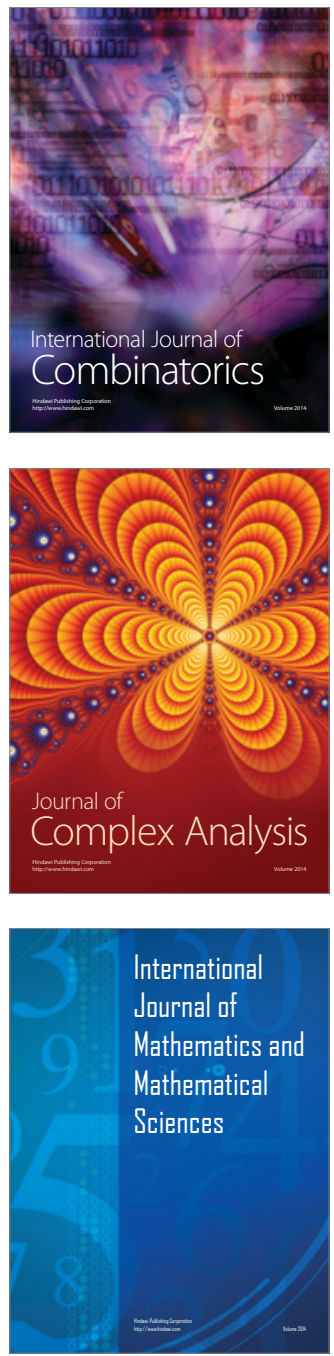
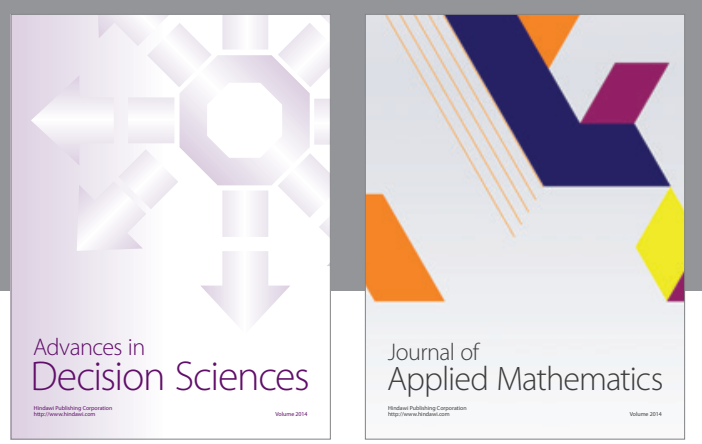

Algebra

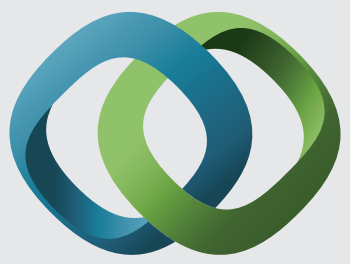

\section{Hindawi}

Submit your manuscripts at

http://www.hindawi.com
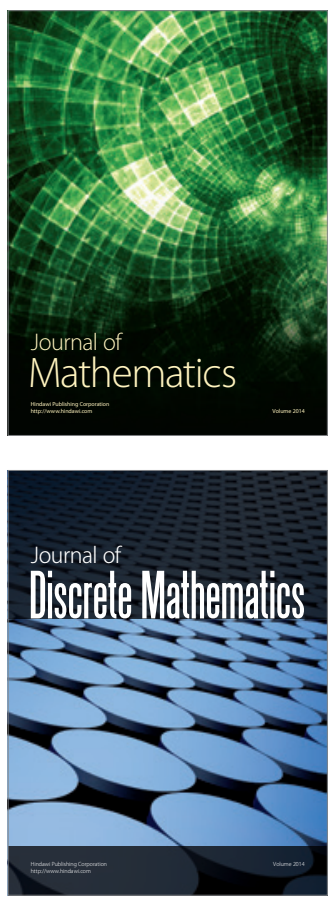

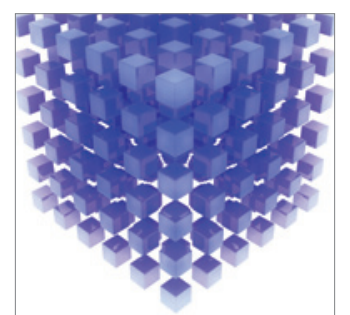

Mathematical Problems in Engineering
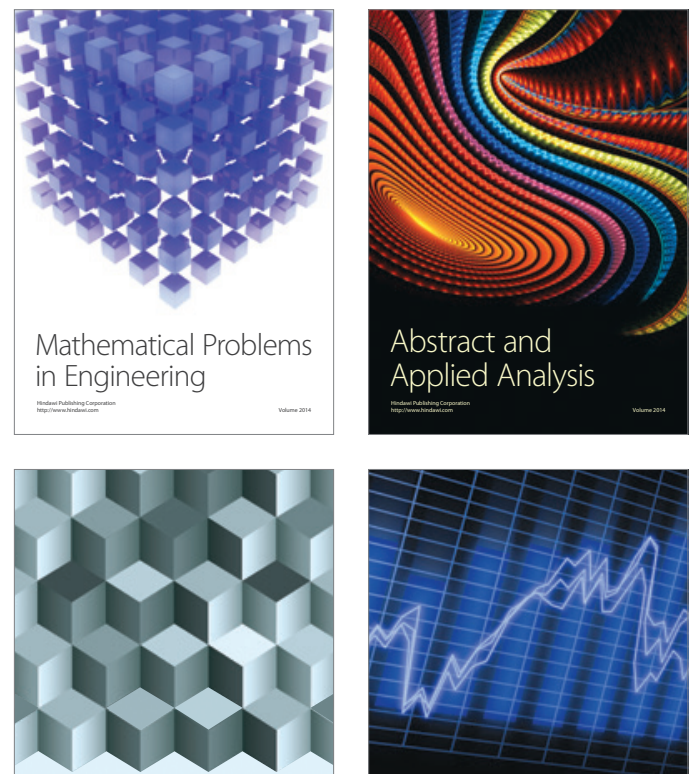

Journal of

Function Spaces

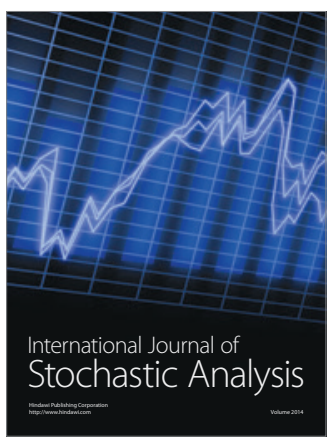

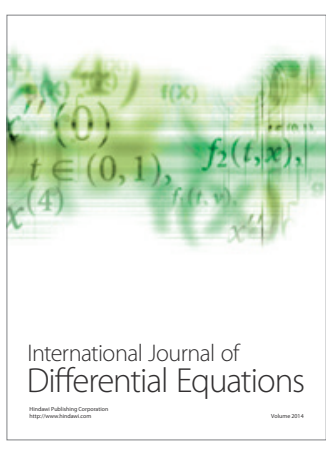
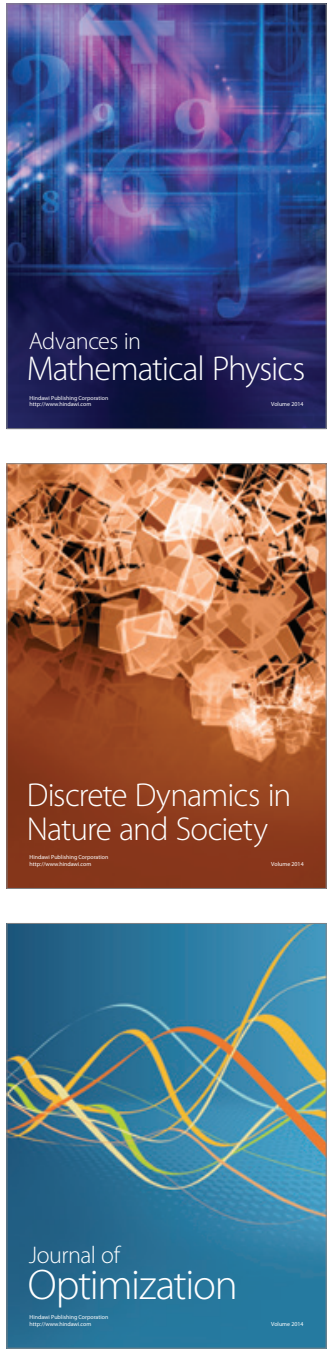